\title{
Le verre et la thermocinétique des matériaux semi-transparents
}

\author{
J. B. Saulnier et J. Martinet \\ Université de Poitiers (UER-ENSMA) et Laboratoire d'Energétique Solaire, rue Guillaume-VII, 86034 Poitiers Cedex, France
}

(Reçu le 10 juillet 1979, révisé le 12 novembre 1979, accepté le 13 novembre 1979)

\begin{abstract}
Résumé. - Après avoir situé le problème des transferts combinés par radiation et conduction dans le cadre général des problèmes liés à l'énergie solaire, la présente étude se présente comme une synthèse des recherches menées jusqu'à présent dans le domaine des milieux semi-transparents et plus particulièrement le verre. Il est particulièrement fait état des méthodes de détermination du champ stationnaire des températures dans les transferts d'abord monomodes (conductif ou radiatif) puis couplés (conductif et radiatif) en renseignant sur l'essentiel des techniques tant anciennes que nouvelles, qui conduisent à cette détermination.
\end{abstract}

Abstract. - We first recall the connection between solar energy and the combined heat transfer by radiation and conduction. We present in this study a synthesis of the different researches about semi-transparent materials, and particularly those concerning glass. We resume the main methods to calculate the steady state temperature fields in problems involving :

- a unique mode of transfer (conduction or radiation).

- the two combined modes (conduction and radiation).

A new technique has been developed concerning the resolution of the equation of conservation of energy (directional discretization of intensities).

\section{Notations.}

$\begin{array}{ll}k, k_{\mathrm{r}} & \begin{array}{l}\text { conductivité thermique phonique, con- } \\ \text { ductivité radiative. }\end{array} \\ \rho c & \text { chaleur volumique. } \\ a & \text { diffusivité thermique. } \\ T & \text { température absolue. } \\ x, z & \text { variables spatiales. } \\ t & \text { variable temporelle. } \\ \theta & \text { variable angulaire. }\end{array}$

$\mu=\cos \theta$,

$\Omega$

$l$

$v$

$\lambda$

$q, q_{\mathrm{r}}, q_{\mathrm{r} \lambda}$

$\mathbf{L}_{v}, \mathbf{L}_{\lambda}$ angle solide.

épaisseur géométrique du mur.

fréquence.

longueur d'onde.

flux calorifique, flux radiatif, flux radiatif monochromatique.

luminances monochromatiques direc- ${ }^{-}$ tionnelles (rapportées à la fréquence ou à la longueur d'onde).

$L_{v}^{0}, L_{\lambda}^{0}, L^{0}$ luminances monochromatiques $\left(L_{v}^{0}, L_{\lambda}^{0}\right)$ ou totale $\left(L^{0}\right)$ du corps noir.

$\chi_{v}, \chi_{\lambda} \quad$ coefficients monochromatiques d'absorption.

$\tau_{\lambda}=\chi_{\lambda} z, \tau_{v}=\chi_{v} z$ épaisseurs optiques monochromatiques. $\tau_{0 \lambda}=\chi_{\lambda} l$ épaisseur optique monochromatique pour $z=l$.

$\tau_{0}=\chi l \quad$ épaisseur optique du mur gris, en $z=l$.

$\xi=\tau / \tau_{0} \quad$ variable spatiale réduite.

$\Phi(\xi), \Theta \quad$ températures réduites.

$n_{v}, n_{\lambda} \quad$ indice de réfraction.

$c_{0} \quad$ vitesse de la lumière dans le vide.

$\sigma, h, k_{\mathrm{B}} \quad$ constantes de Stefan, de Planck, de

Boltzmann.

$h_{\mathrm{s}} \quad$ coefficient d'échange (ou de transfert).

Les grandeurs thermophysiques $k, \rho c, a, n$, seront, dans les équations supposées indépendantes de la température.

1. Introduction. - Quoiqu'essentiellement orientée vers la technique, l'énergie solaire n'est pas sans faire appel aux différents modes de transfert de l'énergie calorifique. C'est en particulier le cas pour les milieux semi-transparents comme le verre dont l'utilisation à basse température est bien connue et comme certains solides ou liquides susceptibles d'être utilisés dans les techniques de stockage.

Dans la plupart des cas, les problèmes qui se posent sont de deux sortes : 
- détermination du champ des températures résultant de conditions d'échauffement données et connues,

- détermination du champ des températures à partir de l'information fournie par l'émission de ces milieux.

L'approche de ces problèmes nécessitait, on le conçoit sans peine, une mise au point des connaissances actuelles dans le domaine des transferts dans les milieux semi-transparents solides mise au point qui a débouché sur une approche nouvelle concernant les discrétisations directionnelle et spatiale d'une part dans le cas des transferts monomodes et d'autre part dans le cas des transferts couplés.

Il est enfin à noter que si la plupart des études entreprises jusqu'à présent sur ce sujet étaient relatives au seul régime stationnaire, la puissance des nouveaux procédés de calcul évoqués dans cette approche (logiciels d'application à la thermique) permet maintenant d'envisager de traiter des problèmes similaires, mais en régime variable. Et si enfin, les exemples choisis ci-après ne concernent que le verre et deux modes de transfert, ces mêmes moyens de calcul permettent dès à présent l'abord de problèmes plus généraux encore qui se posent dans les milieux fluides où interviennent simultanément les trois modes conductif, radiatif et convectif.

2. Rappel des propriétés des matériaux semi-transparents (M.S.T.). - Nous conviendrons de dénommer ainsi les matériaux qui absorbent, émettent, et éventuellement diffusent le rayonnement thermique, dans leur masse, contrairement aux matériaux opaques pour lesquels ces phénomènes se manifestent en surface.

Cette originalité tient au fait que, de par sa structure et la nature de ses constituants, le coefficient monochromatique d'absorption (cf. $\S 4.2 .1$ ) $\chi_{\lambda}$ d'un matériau semi-transparent (coefficient qui est quasi infini pour les matériaux opaques), peut devenir très faible dans les régions spectrales dites de transpa-

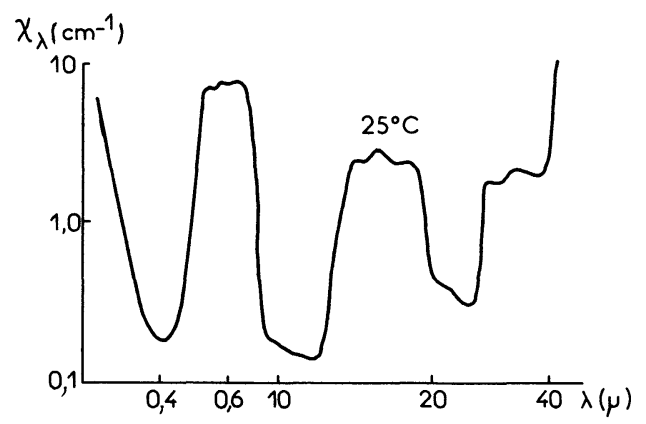

Fig. 1 [1]. - Coefficient monochromatique d'absorption d'un verre à $0,1 \%$ d'oxyde de Cobalt $\mathrm{CoO} .3$ fenêtres de transparence : - la première dans le visible; - la seconde à la limite visible infrarouge; - la troisième dans le proche infrarouge.

[Monochromatic absorption coefficient of a glass with $01 \% \mathrm{CoO}$ : 3 transparency windows : - the first in the visible; - the second in the limit visible infrared; - the third in the near infrared.] rence et par contre conserver des valeurs élevées dans les régions dites d'opacité.

Les fenêtres de transparence peuvent être quelconques, en ce sens qu'elles s'étendent soit dans le domaine visible, soit dans l'infrarouge soit dans les deux régions [1 et 2] (Fig. 1, 2, 3,4).

La présence au sein du M.S.T. d'un transfert radiatif conduit à y définir d'une part le champ de luminance monochromatique directionnelle $\mathbf{L}_{\lambda}$, les champs scalaires de température et de densité de flux d'autre part, enfin les champs liés aux grandeurs

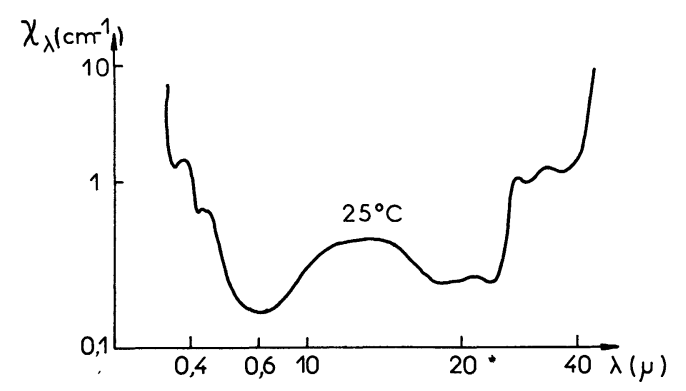

Fig. 2 [1]. - Coefficient monochromatique d'absorption d'un verre à $1 \%$ d'oxyde de fer $\mathrm{F}_{2} \mathrm{O}_{3} .1$ fenêtre étalée dans le visible et le proche infrarouge.

[Monochromatic absorption coefficient of a glass with $1 \% \mathrm{Fe}_{2} \mathrm{O}_{3}$. : 1 transparency window in visible and in infrared.]

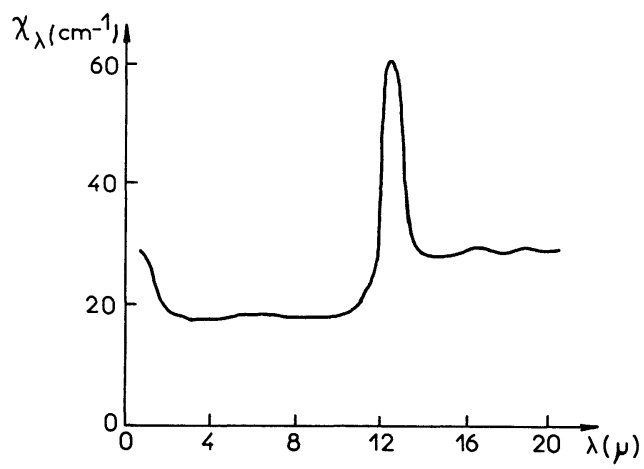

Fig. 3 [2]. - Coefficient monochromatique d'absorption de l'arséniure de silicium. 2 fenêtres dans l'infrarouge.

[Monochromatic absorption coefficient of As $\mathrm{Si}: 2$ transparency windows in the infrared.]

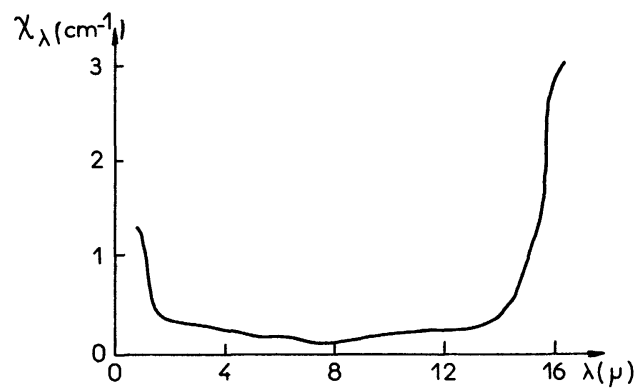

Fig. 4 [2]. - Coefficient monochromatique d'absorption du sulfure de cadmium. 1 fenêtre dans l'infrarouge.

[Monochromatic absorption coefficient of CdS : 1 transparency window in the infrared.] 
thermophysiques (conductivité phonique $k$, diffusivité $a$, chaleur volumique $\rho c$ ) et optiques (indice monochromatique de réfraction $n_{\lambda}$, coefficient monochromatique d'absorption $\chi_{\lambda}$ ), lesquelles peuvent, dans le cas de gradients thermiques modérés, se ramener à des grandeurs scalaires moyennées sur l'espace.

3. Etat actuel des études sur les transferts dans les M.S.T. - Si la thermocinétique des matériaux opaques est aujourd'hui bien connue, son origine remontant à Fourier (1808) [3], il apparaît en revanche que celle des matériaux semi-transparents a connu un départ plus tardif, lié sans doute à l'essence différente des phénomènes mis en jeu, puisque c'est seulement en 1903 que Schuster [4] donnait le premier point de vue des astrophysiciens sur l'influence du rayonnement dans un transfert de chaleur.

Dès 1920 le problème prenait un caractère industriel, puisqu'était alors reconnue la nécessité de prendre en compte, dans le bilan thermique au sein des chaudières [5], le transfert par rayonnement au travers des gaz de combustion $\left(\mathrm{CO}_{2}, \mathrm{H}_{2} \mathrm{O}\right)$.

Preston [6] arrivait, vers 1940 à des conclusions analogues et ce, bien que ses travaux eussent trait à un tout autre matériau, à savoir le verre.

La complexité du phénomène, et en particulier les effets des frontières et des dépendances spectrales des propriétés optiques du matériau en rendaient difficile la description mathématique; aussi était-il naturel que les premières tentatives aient eu tendance à schématiser le problème :

- Czerny et Genzel [7], Geffcken [8] ont, par exemple, traité le transfert radiatif pur dans un milieu gris ou non gris, tout en tenant compte de la nature diffuse du rayonnement : il est alors apparu, qu'à condition de considérer une grande épaisseur de verre, le transfert radiatif s'apparentait au transfert conductif, en ce sens que le flux de chaleur radiatif restait proportionnel au gradient de température, ce qui a été à l'origine de l'introduction de la notion de conductivité radiative (cf. $\S 4.2 .3 .4$ ), laquelle est directement liée au coefficient d'absorption [7].

- L'influence de la frontière du milieu a été examinée par Geffcken [9] ainsi que par Walter, Dorr et Eller [10] (surfaces limites noires). Ils ont montré, en linéarisant l'équation intégrodifférentielle du transfert (ce qui suppose de faibles gradients), que la présence simultanée des deux modes de transfert au sein d'un matériau gris, conduisait à un champ de température stationnaire non linéaire dans le problème du mur fini où l'on impose deux températures différentes sur les frontières. Tout en linéarisant également l'équation du transfert, Poltz [11] a introduit l'effet de réflexion partielle aux limites, ce qui était à l'origine d'un terme correctif dans les résultats précédents.
- Plus récemment Chui et Gardon [12] ont montré les conséquences de la nature des conditions imposées aux limites (flux, températures) d'un mur fini, sur l'allure du champ stationnaire de température : la forme des courbes n'est alors pas unique, et leur courbure dépend, entre autres, fortement de la valeur du coefficient de transfert $h_{\mathrm{s}}$ entre le mur (ici de verre) et son environnement. La figure 5 montre par exemple l'écart entre les champs thermiques dans le cas de températures imposées aux limites (courbe $A$ : $h_{\mathrm{s}}$ infini) et de flux imposé (courbe $B$ :

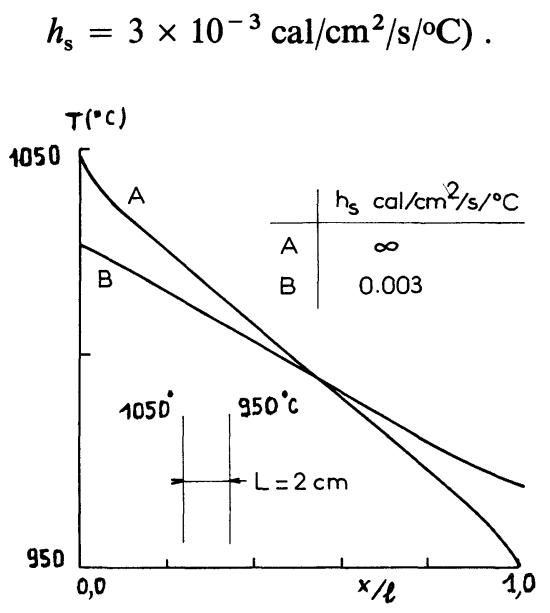

Fig. 5 [12]. - Champ de température stationnaire dans un mur gris (verre gris $\chi=5 \mathrm{~cm}^{-1}, k=3 \times 10^{-3} \mathrm{cal} / \mathrm{cm} /{ }^{\circ} \mathrm{C} / \mathrm{s}$ ).

[Steady state field of temperature in a gray wall (gray glass : $\left.\chi=5 \mathrm{~cm}^{-1}, k=3 \times 10^{-3} \mathrm{cal} / \mathrm{cm} /{ }^{\circ} \mathrm{C}\right)$.]

C'est en fait le développement des ordinateurs qui a permis de résoudre les équations dans le cas le plus général d'une dépendance spectrale marquée, d'épaisseurs quelconques, voire de régimes transitoires. On a ainsi traité le problème direct de la détermination numérique des champs de température et de flux pour des valeurs bien définies des paramètres précédents, par des moyens conventionnels $[2,13,14]$ ou plus actuels [38].

En matière de recherche expérimentale, il est apparu rapidement indispensable, dès lors que l'hypothèse du matériau gris n'était plus retenue, d'obtenir des informations sur les variations du facteur d'absorption tant avec la longueur d'onde, qu'avec la température. Cela a fait l'objet des travaux de Genzel [15], Neuroth [16, 17], Grove et Jellyman [18], Coenen [1], Blazek [19], Wedding [20], et plus récemment, de ceux de $M$. Traverse [21], lesquels tout en résumant l'évolution des techniques des auteurs précédents, présentent une méthode originale : l'étude se place à haute température et l'échantillon de verre en fusion est soutenu par une légère surpression gazeuse, ce qui permet de s'affranchir d'une part des problèmes de réflexion du faisceau de mesure sur les hublots et d'autre part de l'attaque et la dégradation de ces derniers par les ions alcalins du verre fondu. 
Il était séduisant, dès lors que le transfert radiatif était assimilable à un phénomène de diffusion [7], [8], d'où une conductivité propre, de déduire celle-ci des mesures de son coefficient d'absorption monochromatique [1], [18], [19]. Plus encore, on a longtemps pensé atteindre le transfert global grâce à la somme algébrique des conductivités phonique et radiative et ce quelle que soit la géométrie du milieu. C'est ainsi que nombre d'expérimentateurs ont tenté de mesurer, par des méthodes appropriées (signal périodique radial [22], axial [23], [24], cylindre creux [25], [26], fil chaud [27]) tant la conductivité globale que la diffusivité associée. C'était en fait oublier l'hypothèse du milieu semi-infini [7], [8] qui conduit, entre autres, à la notion de conductivité radiative, mais qui n'est pas compatible avec la présence de frontières à de courtes distances de la zone de mesure, comme c'était le cas dans la plupart des expériences précédentes. Il en résulte en particulier, que la notion algébrique de conductivité globale n'est pas une grandeur intrinsèque caractéristique du matériau : elle dépend de la géométrie, voire de la méthode de mesure, ainsi que le montrent le tableau I [28] et la figure 6 [29]. A l'appui de ces observations s'ajoutent ici les résultats de Gardon [30], lequel, poursuivant

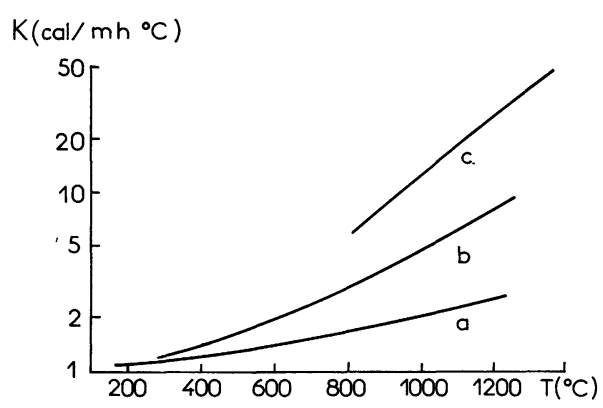

Fig. 6. - Influence de l'épaisseur de l'échantillon sur la valeur mesurée de la conductivité. a) [26] Eckhardt : épaisseur $9 \mathrm{~mm}$; b) [25] Eligehausen : épaisseur $17,5 \mathrm{~mm}$; c) [22] Van Zee und Babcock : épaisseur $165 \mathrm{~mm}$.

[Variation of the measured value of the conductivity with the thickness. $a$ ) [26] Eckhardt : thickness $9 \mathrm{~mm} ; b$ ) [25] Eligehausen : thickness $17.5 \mathrm{~mm}$; c) [22] Van Zee and Babcock : thickness $165 \mathrm{~mm}$. les travaux de Czerny, a montré que même pour un milieu fini, il était possible de calculer une conductivité globale (qualifiée d'apparente), mais qui n'en demeurait pas moins fonction de l'épaisseur du matériau. A une température donnée, cette grandeur se trouve comprise (Fig. 7) entre la conductivité phonique (c'est-à-dire correspondant à une épaisseur tendant vers zéro) et la conductivité du milieu infini (calculée par Czerny).

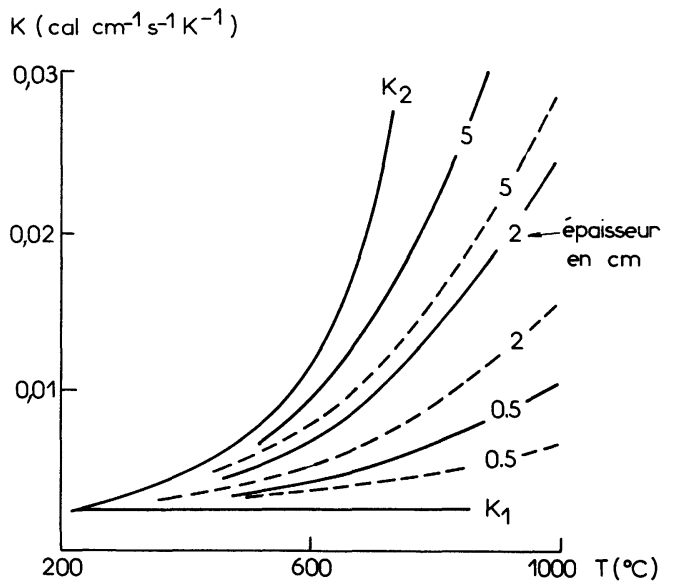

Fig. 7. - Conductivité apparente calculée par simulation unidimensionnelle en régime stationnaire de verres en fonction de la température, de l'épaisseur et des conditions aux limites. --- thermodes non mouillées par le verre; $\longrightarrow$ thermodes réfractaires mouillées par le verre; $k_{1}$ conductivité phonique; $k_{2}$ expression de Czerny.

[Apparent glass conductivity, calculated by a monodimensional steady state simulation, as a function of temperature, thickness, and boundary conditions. --- wetted thermodes; - refractory not wetted thermodes; $k_{1}$ phonic conductivity; $k_{2}$ Czerny expression.]

Les derniers résultats expérimentaux sont à mettre en liaison avec les résolutions numériques, déjà citées, des problèmes de couplage. Il s'agit en effet de méthodes de mesure du champ de température soit directement par interférométrie [31] ou absorption d'un rayonnement laser [32], soit indirectement [33], en utilisant des mesures spectrales directionnelles du

Tableau I. - Influence de l'épaisseur de l'échantillon sur la valeur mesurée de la diffusivité du verre blanc $\left(\mathrm{cm}^{2} / \mathrm{s}\right)$. [Measured value of the conductivity of white glass as a function of the thickness.]

\begin{tabular}{|c|c|c|c|c|c|c|c|c|}
\hline \multirow{2}{*}{$\begin{array}{l}\text { Température } \\
\left({ }^{\circ} \mathrm{C}\right)\end{array}$} & \multicolumn{8}{|c|}{ Epaisseur du verre (en inches) } \\
\hline & 0,25 in. & $1 \mathrm{in.}$ & & 3 in. & 3,5 in. & $6 \mathrm{in}$. & 8 in. & $12 \mathrm{in.}$ \\
\hline 500 & env. $0,4 \times 10^{-2}$ & & 2 & $\times 10^{-2}$ & $2 \times 10^{-2}$ & $2 \times 10^{-2}$ & .. & $2 \times 10^{-2}$ \\
\hline 750 & & $1 \times 10^{-2}$ & 2,8 & $3 \times 10^{-2}$ & $2,8 \times 10^{-2}$ & & $3,8 \times 10^{-2}$ & $4,2 \times 10^{-2}$ \\
\hline 900 & & & 3,5 & $5 \times 10^{-2}$ & $5 \times 10^{-2}$ & & $7,4 \times 10^{-2}$ & $10 \times 10^{-2}$ \\
\hline 1000 & & & 5 & $\times 10^{-2}$ & 5,5 à $8 \times 10^{-2}$ & & $9 \times 10^{-2}$ & $\times 10^{-2}$ \\
\hline 1100 & & $2 \times 10^{-2}$ & 6,5 & $5 \times 10^{-2}$ & $10 \times 10^{-2}$ & $12 \times 10^{-2}$ & $12,5 \times 10^{-2}$ & $\times 10^{-2}$ \\
\hline 1200 & env. 4 & & 10 & $\times 10^{-2}$ & $13 \times 10^{-2}$ & & & \\
\hline 1300 & & & 14 & $\times 10^{-2}$ & $\times 10^{-2}$ & & & \\
\hline
\end{tabular}


flux émis, lesquelles sont ensuite introduites dans l'équation de l'énergie, en vue de déterminer, par inversion de cette dernière, le profil des températures.

4. Les transferts monomodes conductif et radiatif. Afin de mieux percevoir la contribution du couplage (cf. § 5) nous rappellerons tout d'abord quelques résultats concernant les transferts où intervient un seul mécanisme (rayonnement ou conduction) et prendrons, à titre de comparaison le même exemple dans tous les cas, à savoir celui d'un mur fini ayant atteint un état stationnaire, lorsque, partant d'une répartition initiale uniforme nulle, on impose les températures $T_{0}$ et $T_{1}\left(T_{1}>T_{0}\right)$ sur les frontières $x=0$ et $x=l$ : nous situerons alors nos résultats par rapport à ceux trouvés dans la littérature, et discuterons les avantages respectifs des différentes approches utilisées.

4.1 LE TRANSFERT CONDUCTIF. - Le champ spatiotemporel de température $T(x, t)$ est toujours régi par l'équation de conservation :

$$
\operatorname{div} \mathbf{q}+\rho c \frac{\partial T}{\partial t}(x, t)=0
$$

où $\mathbf{q}$ désigne le vecteur flux calorifique donné ici par la loi de Fourier :

$$
\mathbf{q}=-k \operatorname{grad} T .
$$

Il s'ensuit que le champ stationnaire correspondant à l'exemple cité plus haut est linéaire de $T_{0}$ à $T_{1}$.

4.2 Le TRANSFert RADIATIF. - Dans un milieu inactif tel que le vide, la luminance directionnelle monochromatique ou totale définie comme le quotient du flux monochromatique ou total (par unité de surface normale à la direction de propagation et par unité d'angle solide) se conserve tout au long du trajet de l'énergie, obéit à la loi de Planck (pour autant que la source puisse être assimilée à un corps noir) et se comporte comme une grandeur directionnelle isotrope et homogène. Dès lors que l'on aborde un matériau susceptible d'interaction avec le rayonnement (absorption, émission induite ou spontanée), se manifestent des modifications portant sur l'amplitude, les distributions spatiale et spectrale ainsi que l'homogénéité de la luminance.

4.2.1 Emission. Absorption. Equation de la luminance. - Le comportement radiatif d'un milieu actif est gouverné à la fois par les lois de l'émission et de l'absorption de l'énergie, lesquelles sont impliquées dans l'équation de la luminance (1) ci-dessous.

Considérons un milieu semi-infini dans la direction $x^{\prime} x$. La variation de luminance à l'abscisse $x$, sur un trajet $\mathrm{d} z$ qui fait un angle $\theta$ avec $x^{\prime} x(\mu=\cos \theta)$ est donnée par l'expression différentielle bien connue de l'équation de la luminance :

$$
\mu \mathrm{d} \mathbf{L}_{v}=-\chi_{v}\left(\mathbf{L}_{v}-S_{v}\right) \mathrm{d} z
$$

qui regroupe les contributions locales

$$
\begin{aligned}
& \text { - de l'absorption : - } \chi_{v} \mathbf{L}_{v} \mathrm{~d} z, \\
& \text { - de l'émission : } \chi_{v} S_{v} \mathrm{~d} z,
\end{aligned}
$$

où apparaissent en particulier le coefficient monochromatique d'absorption $\chi_{v}$ (lequel tient compte à la fois de l'absorption vraie et de l'émission induite) ainsi que la fonction source $S_{v}$.

Le problème reste alors à préciser la fonction $S_{v}$ (dimensionnée à une luminance), à résoudre l'équation (1), et, pour le thermicien, d'en déduire le champ des températures, voire celui des flux. Pour ce qui est de la fonction source, seront retenues uniquement les circonstances où s'applique le principe de l'équilibre thermodynamique local (excluant par exemple les phénomènes de diffusion, d'anisotropie, les phénomènes très courts ou très intenses pour lesquels les propriétés statistiques locales soit n'entrent pas en jeu, soit sont perturbées au point de ne plus rester boltzmanniennes $[34,35]$ ) et nous en déduirons par un calcul classique portant sur les probabilités de transitions que $S_{v}$ n'est autre que la fonction de Planck :

$$
S_{v}=L_{v}^{0}=\frac{2 h v^{3}}{c^{2}} \frac{1}{\exp \frac{h v}{k_{\mathrm{B}} T}-1}
$$

Une première intégration de (1) conduit alors à la solution formelle suivante :

$$
\begin{aligned}
& \mathbf{L}_{v}\left(\tau_{v}, \mu\right)=\mathbf{L}_{v}(0, \mu) \exp \left(-\frac{\tau_{v}}{\mu}\right)+ \\
& \quad+\int_{0}^{\tau_{v}} L_{v}^{0}\left(\tau_{v}^{*}\right) \exp \left(-\frac{\tau_{v}-\tau_{v}^{\prime}}{\mu}\right) \frac{\mathbf{d} \tau_{v}^{\prime}}{\mu}
\end{aligned}
$$

où l'on a posé $\tau_{v}=\chi_{v} x$.

L'exemple choisi du mur fini conduit à écrire (2) en tenant compte de ce qu'aux limites $x=0$ et $x=l$ la luminance monochromatique (rapportée ici à $\lambda$ ) prend les valeurs bien déterminées $\mathbf{L}_{\lambda}(0, \mu)$ et $\mathbf{L}_{\lambda}\left(\tau_{0 \lambda}, \mu\right)$, lesquelles sont des fonctions des températures des frontières, ainsi que de leur nature (diffuses, noires, semi-transpareñtes...) et où $\tau_{0 \lambda}$ n'est autre que l'épaisseur optique monochromatique du matériau $\chi_{\lambda} l$.

Notant alors $\mathbf{L}_{\lambda}^{+}\left(\tau_{\lambda}, \mu\right)$ et $\mathbf{L}_{\lambda}^{-}\left(\tau_{\lambda},-\mu\right)$ les luminances correspondant à des radiations se propageant respectivement vers l'hémisphère $x>0$ et l'hémisphère $x<0$, la relation (2) se décompose comme suit : 


$$
\begin{gathered}
\mathbf{L}_{\lambda}^{+}\left(\tau_{\lambda}, \mu\right)=\mathbf{L}_{\lambda}^{+}(0, \mu) \exp \left(-\frac{\tau_{\lambda}}{\mu}\right)+\int_{0}^{\tau_{\lambda}} \mathbf{L}_{\lambda}^{0}\left(\tau_{\lambda}^{*}\right) \exp \left(-\frac{\tau_{\lambda}-\tau_{\lambda}^{*}}{\mu}\right) \frac{\mathrm{d} \tau_{\lambda}^{*}}{\mu} \\
\mathbf{L}_{\lambda}^{+}\left(\tau_{\lambda},-\mu\right)=\mathbf{L}_{\lambda}^{-}\left(\tau_{0 \lambda},-\mu\right) \exp \left(-\frac{\tau_{0 \lambda}-\tau_{\lambda}}{\mu}\right)+\int_{\tau_{0 \lambda}}^{\tau_{\lambda}} \mathbf{L}_{\lambda}^{0}\left(\tau_{\lambda}^{*}\right) \exp \left(-\frac{\tau_{\lambda}^{*}-\tau_{\lambda}}{\mu}\right) \frac{\mathrm{d} \tau_{\lambda}}{\mu}
\end{gathered}
$$

avec $\mu>0$ dans les deux cas.

La résolution de (3a) et (3b) nécessite la connaissance des valeurs limites $\mathbf{L}_{\lambda}^{-}(0, \mu)$ et $\mathbf{L}_{\lambda}^{+}\left(\tau_{0 \lambda},-\mu\right)$; mais plus encore, la fonction de Planck qui apparaît dans l'intégrandum dépend, à travers $L^{0}\left(\tau_{\lambda}^{*}\right)$ et de façon implicite, de la température $T(x)$ sur laquelle nous n'avons jusqu'à présent aucune information. Cette variable nécessite l'adjonction d'une équation supplémentaire, que nous obtenons en écrivant la conservation de l'énergie :

$$
\operatorname{div} q_{\mathrm{r}}(x)+\rho c \frac{\partial T}{\partial t}(x, t)=0
$$

où $q_{\mathrm{r}}(x)$ figure le flux radiatif total à l'abscisse $x$.

4.2.2 Le flux radiatif et sa divergence. - Son expression n'est autre que :

$$
q_{\mathrm{r}}(x)=\int_{0}^{\infty} q_{\mathrm{r} \lambda}\left[\tau_{\lambda}(x)\right] \mathrm{d} \lambda
$$

dont la divergence qui s'écrit :

$$
\operatorname{div} q_{\mathrm{r}}(x)=\int_{0}^{\infty} \chi_{\lambda} \frac{\mathrm{d} q_{\mathrm{r} \lambda}\left(\tau_{\lambda}\right)}{\mathrm{d} \tau_{\lambda}} \mathrm{d} \lambda
$$

est chiffrable dès lors qu'est connu le terme différentiel du second membre. Lié au champ des luminances monochromatiques par :

$$
q_{\mathrm{r} \lambda}\left(\tau_{\lambda}\right)=\int_{4 \pi} \mathbf{L}_{\lambda}\left(\tau_{\lambda}, \mu\right) \mu \mathrm{d} \Omega=2 \pi\left[\int_{0}^{1} \mathbf{L}_{\lambda}^{+}\left(\tau_{\lambda}, \mu\right) \mu \mathrm{d} \mu-\int_{0}^{1} \mathbf{L}_{\lambda}^{-}\left(\tau_{\lambda},-\mu\right) \mu \mathrm{d} \mu\right],
$$

le flux monochromatique radiatif s'écrit encore, compte tenu de $(3 a)$ et $(3 b)$ :

$$
\begin{aligned}
\frac{q_{\mathrm{r} \lambda}\left(\tau_{\lambda}\right)}{2 \pi}=\int_{0}^{1} \mathbf{L}_{\lambda}^{+}(0, \mu) \exp \left(-\frac{\tau_{\lambda}}{\mu}\right) \mu \mathrm{d} \mu-\int_{0}^{1} \mathbf{L}_{\lambda}^{-}\left(\tau_{0 \lambda},-\mu\right) \exp \left(-\frac{\tau_{0 \lambda}-\tau}{\mu}\right) \mu \mathrm{d} \mu+ \\
\quad+\int_{0}^{\tau_{\lambda}} L_{\lambda}^{0}\left(\tau_{\lambda}^{*}\right) E_{2}\left(\tau_{\lambda}-\tau_{\lambda}^{*}\right) \mathrm{d} \tau_{\lambda}^{*}-\int_{\tau_{\lambda}}^{\tau_{0 \lambda}} L_{\lambda}^{0} E_{2}\left(\tau_{\lambda}^{*}-\tau_{\lambda}\right) \mathrm{d} \tau_{\lambda}^{*},
\end{aligned}
$$

expression où sont introduites les fonctions intégro-exponentielles d'ordre $n=2$ et dont la définition générale est :

$$
E_{n}(t)=\int_{0}^{1} \exp \left(-\frac{t}{\mu}\right) \mu^{n-2} \mathrm{~d} \mu .
$$

Dérivant (7) par rapport à $\tau_{\lambda}$ il vient alors :

$$
\begin{array}{r}
\frac{1}{2 \pi} \frac{\mathrm{d} q_{\mathrm{r} \lambda}\left(\tau_{\lambda}\right)}{\mathrm{d} \tau_{\lambda}}=-\int_{0}^{1} \mathbf{L}_{\lambda}^{+}(0, \mu) \exp \left(-\frac{\tau_{\lambda}}{\mu}\right) \mathrm{d} \mu-\int_{0}^{1} \mathbf{L}_{\lambda}^{-}\left(\tau_{0 \lambda},-\mu\right) \exp \left(-\frac{\tau_{0 \lambda}-\tau_{\lambda}}{\mu}\right) \mathrm{d} \mu- \\
-\int_{0}^{\tau_{0} \lambda} L_{\lambda}^{0}\left(\tau_{\lambda}^{*}\right) E_{1}\left(\left|\tau_{\lambda}^{*}-\tau_{\lambda}\right|\right) \mathrm{d} \tau_{\lambda}^{*}+2 L_{\lambda}^{0}\left(\tau_{\lambda}\right) .
\end{array}
$$


4.2.3 Les méthodes de résolution du problème du mur fini. - Nous ne traiterons ici que le cas des frontières noires et d'un coefficient d'absorption gris $\left(\chi_{\lambda}\right.$ indépendant de $\left.\lambda\right)$; l'étude de la dépendance spectrale sera exposée en abordant le couplage radiatif conductif (cf. §5).

Nous avons développé et comparé trois méthodes d'approche :

- la première classique qui fait appel aux ressources les plus poussées de l'analyse mathématique (équation de Fredholm);

- la seconde, plus originale, qui implique la discrétisation directionnelle des luminances et qui conduit au calcul simultané des champs de luminance et de température ;

- la dernière, qui découle de l'analyse nodale, dont l'application aux matériaux partiellement absorbants constitue un aspect nouveau. Elle s'étend, de plus, aisément au transfert couplé (cf. §5).

Ces deux dernières nous ont permis d'appliquer à la thermique des matériaux semi-transparents les moyens de calcul encore peu connus que sont les logiciels.

Nous rappellerons enfin pour mémoire la solution approchée de la diffusion, dont la validité sera plus particulièrement discutée au $§ 5.4$.

4.2.3.1 Intégration de l'équation de Fredholm.Compte tenu des hypothèses (frontières noires, mur gris), l'équation de conservation de l'énergie prend la forme :

$n^{2} \sigma T_{0}^{4} E_{2}(\tau)+n^{2} \sigma T_{1}^{4} E_{2}\left(\tau_{0}-\tau\right)$

$-2 n^{2} \sigma T^{4}(\tau)+\int_{0}^{\tau_{0}} n^{2} \sigma T^{4}\left(\tau^{*}\right) E_{1}\left(\left|\tau-\tau^{*}\right|\right) \mathrm{d} \tau^{*}=0$.

Après avoir effectué les changements de variable

$$
\xi=\frac{x}{l}=\frac{\tau}{\tau_{0}} \quad \text { et } \quad \Phi(\xi)=\frac{T^{4}(\xi)-T_{1}^{4}}{T_{0}^{4}-T_{1}^{4}}
$$

le champ de température ainsi réduit est solution de l'équation de Fredholm de première espèce :

$2 \Phi(\xi)=E_{2}\left(\xi \tau_{0}\right)+\tau_{0} \int_{0}^{1} \Phi(u) E_{1}\left(\tau_{0}|\mu-\xi|\right) \mathrm{d} u ，$

laquelle présente une singularité, qui est celle de $E_{1}$ à l'origine. Diverses techniques de résolution de ce type d'équations se trouvent développées dans la littérature : méthode itérative [36], des moindres carrés [37]. Nous avons pour notre part [38] utilisé une voie classique consistant à lever la singularité par une intégration par parties, à tabuler la fonction $E_{2}$ (méthode de Romberg) à discrétiser la fonction $\Phi$ enfin à résoudre par la méthode de GaussSeidel le système linéaire obtenu; le FORTRAN a été retenu ici comme langage de travail. Dès lors que l'on connaissait $T(\tau)$, il était possible de décrire, par l'intermédiaire de $(3 a)$, (3b), (5) et (7) le champ de luminance, ainsi que celui des flux monochromatique et total.

4.2.3.2 Discrétisation directionnelle. - De par l'allure des intégrales définissant le flux radiatif (cf. § 4.2.2), il est avantageux $[13,38,39]$ de le développer suivant une somme gaussienne [40], selon :

$$
\begin{aligned}
& q_{\mathrm{r}}(x)=2 \pi \int_{-1}^{+1} \mathbf{L}(x, \mu) \mu \mathrm{d} \mu \\
&=2 \pi \sum_{i=1}^{m} \omega_{i} \mu_{i} \mathbf{L}\left(x, \mu_{i}\right)
\end{aligned}
$$

où les arguments $\mu_{i}$ (opposés 2 à 2) et les poids $\omega_{i}$ (égaux deux à deux) sont connus dès lors qu'est fixé l'ordre $m=2 m^{\prime}$ de la somme gaussienne. La propagation du rayonnement étant ainsi discrétisée selon $2 \mathrm{~m}^{\prime}$ directions, nous noterons $\mathbf{L}_{i}(x)=\mathbf{L}\left(x, \mu_{i}\right)$ la' luminance directionnelle totale, dans l'une quelconque de ces directions. Dans ces conditions les équations de la luminance et de la conservation de l'énergie prennent, dans le cas $m=4\left(\mu_{1}=-\mu_{4}>0\right.$, $\mu_{2}=-\mu_{3}>0$ ) la forme :

$$
\begin{gathered}
\frac{\mu_{i}}{x} \frac{\mathrm{dL}_{i}(x)}{\mathrm{d} x}=\frac{n^{2} \sigma T^{4}(x)}{\pi}-\mathbf{L}_{i}(x) \quad i=1,2,3,4 \\
\sum_{i} \omega_{i} \mu_{i} \frac{\mathrm{d} \mathbf{L}_{i}(x)}{\mathrm{d} x}=0
\end{gathered}
$$

ou encore :

$$
\begin{gathered}
\frac{\mu_{i}}{x} \frac{\mathrm{d} \mathbf{L}_{i}(x)}{\mathrm{d} x}=\frac{1}{2} \sum_{j} \omega_{j} \mathbf{L}_{j}(x)-\mathbf{L}_{i}(x) \quad i=1,2,3,4 \\
\frac{n^{2} \sigma T^{4}(x)}{\pi}=\frac{1}{2} \sum_{j} \omega_{j} \mathbf{L}_{j}(x) .
\end{gathered}
$$

Deux voies s'offrent alors pour l'intégration de ce système : l'une, classique procède par itérations [13], l'autre, que nous avons développée récemment [38] fait appel à des techniques d'optimisation que l'on peut résumer ainsi : le système (13) des équations différentielles couplées en $\mathbf{L}_{i}$ ne peut s'intégrer immédiatement par exemple par la méthode de Runge-Kutta, car sur les 4 valeurs à la limite $x=0$, deux seules sont connues, $\mathbf{L}_{1}(0)=\mathbf{L}_{2}(0)=\frac{n^{2} \sigma T_{0}^{4}}{\pi}$ et deux sont inconnues, $p_{1}=\mathbf{L}_{3}(0)$ et $p_{2}=\mathbf{L}_{4}(0)$.

Une incrémentation adéquate (méthode SIMPLEX par exemple) agit [38] sur $p_{1}$ et $p_{2}$ de telle sorte que la fonction critère 
Tableau II. - Expression des conductances radiatives dans un mur fini de matériau semi-transparent.

[Expression of the radiative conductances in a semi transparent wall.]

\begin{tabular}{|l|c|c|c|}
\hline \multicolumn{2}{|c|}{ Nouds } & Conductance $/ n^{2} \sigma$ & Expression \\
\hline $\begin{array}{l}\text { Frontière 0 } \\
\text { Frontière 1 }\end{array}$ & 0 & $b_{0} b_{1}$ & $2 E_{3}(\chi l)$ \\
\hline $\begin{array}{l}\text { Matériau } \\
\text { Frontière 0 }\end{array}$ & $\begin{array}{c}\left(i+\frac{1}{2}\right) \Delta x \\
0\end{array}$ & $m_{i} b_{0}$ & $2\left\{E_{3}(\chi i \Delta x)-E_{3}(\chi(i+1) \Delta x)\right\}$ \\
\hline $\begin{array}{l}\text { Matériau } \\
\text { Frontière 1 }\end{array}$ & $\begin{array}{c}\left(i+\frac{1}{2}\right) \Delta x \\
l\end{array}$ & $m_{i} b_{1}$ & $2\left\{E_{3}\left(\chi(l-(i+1) \Delta x)-E_{3}(\chi(l-i \Delta x))\right\}\right.$ \\
\hline $\begin{array}{l}\text { Matériau } \\
\text { Matériau }\end{array}$ & $\begin{array}{l}\left(i+\frac{1}{2}\right) \Delta x \\
\left(j+\frac{1}{2}\right) \Delta x\end{array}$ & $m_{i} m_{j}$ & $\begin{array}{r}2\left\{E_{3}(\chi(j+i-1) \Delta x)+\right. \\
+E_{3}(\chi(j-i-1) \Delta x) \\
\left.2 E_{3}(\chi(j-i) \Delta x)\right\}\end{array}$ \\
\hline
\end{tabular}

$$
\begin{aligned}
J\left(p_{1}, p_{2}\right)=\left[\mathbf{L}_{3}(x=l)\right. & \left.-\frac{n^{2} \sigma T_{1}^{4}}{\pi}\right]^{2}+ \\
& +\left[\mathbf{L}_{4}(x=l)-\frac{n^{2} \sigma T_{1}^{4}}{\pi}\right]^{2}
\end{aligned}
$$

atteigne une valeur minimale. Le champ $T(x)$ correspondant aux valeurs $p_{1}$ et $p_{2}$ ainsi déterminées, est alors tracé par l'équation (14) et constitue la solution du problème.

Les avantages de cette approche, au demeurant nouvelle, proviennent d'une part de la facilité du codage du problème (à l'aide du logiciel CSMP), et d'autre part de ce que les champs de luminances et de température sont obtenus simultanément, lors de l'intégration des équations (13) et (14).

4.2.3.3 Discrétisation spatiale. - Cette analyse bien connue des problèmes de thermique provient soit de l'écriture discrétisée (par rapport aux variables spatiales) des équations aux dérivées partielles décrivant le phénomène, soit de la méthode nodale. Nous avons retenu cette dernière approche et fait appel au logiciel SINDA, utilisé jusqu'ici essentiellement dans le cadre de projets à caractère spatial. Nous avons pour cela décomposé le mur en un nombre $N$ de nœuds équidistants $(\Delta x=l / N)$ situés au centre d'éléments de volume supposés isothermes et de capacité $C_{\mathrm{i}}$ (Fig. 14). Les conductances entre les différents nœuds sont ici essentiellement de type radiatif et sont établies en [41]. Elles font appel à 3 expressions différentes suivant la nature des nœuds mis en jeu (ainsi que le résume le tableau II)

entre élément $i$ du milieu et la frontière $b_{j}: n^{2} \sigma m_{i} b_{j}$, entre éléments $i$ et $j$ du milieu : $\quad n^{2} \sigma m_{i} m_{j}$. entre surfaces frontières $n^{2} \sigma b_{0} b_{1}$

Outre le fait que nous avons étendu ici le domaine d'application du logiciel SINDA aux matériaux semitransparents, l'intérêt de cette approche réside dans l'ouverture vers les régimes transitoires, dont la mise en équation conduit à résoudre le système d'équations différentielles :

$$
\begin{aligned}
C_{i} \dot{T}_{i}=n^{2} \sigma m_{i} b_{0}\left(T_{0}^{4}\right. & \left.-T_{i}^{4}\right)+n^{2} \sigma m_{i} b_{1}\left(T_{1}^{4}-T_{i}^{4}\right) \\
& +\sum_{j} n^{2} \sigma m_{i} m_{j}\left(T_{j}^{4}-T_{i}^{4}\right)
\end{aligned}
$$

lequel, dans le cas présent (stationnaire) se ramène simplement à un système algébrique.

De plus, ce traitement par la méthode nodale offre l'avantage de se prêter aisément à l'introduction du phénomène de conduction (cf. $\S 5.3$ ).

4.2.3.4 L'approximation de la diffusion et la conductivité radiative. - Elle découle de l'hypothèse suivante, à savoir que l'épaisseur optique monochromatique du milieu est très grande devant l'unité, et ce, pour toutes les longueurs d'ondes impliquées dans le transfert. Il s'ensuit que le rayonnement ne peut alors se propager loin, puisqu'étant absorbé sur un faible trajet. Ainsi la contribution à l'énergie présente dans un volume élémentaire ne provient-elle que de son proche environnement; il est intuitif dans ces conditions que le flux radiatif ne dépend que des propriétés locales, comme en conduction pure, l'énergie diffusant alors de proche en proche.

Il est alors justifié [5] de développer la fonction source autour de $\tau_{\lambda}$ selon :

$$
\begin{aligned}
L_{\lambda}^{0}\left(\tau_{\lambda}^{*}, \mu\right)=L_{\lambda}^{0}\left(\tau_{\lambda}, \mu\right) & +\left(\tau_{\lambda}^{*}-\tau_{\lambda}\right) \frac{\mathrm{d} L_{\lambda}^{0}\left(\tau_{\lambda}, \mu\right)}{\mathrm{d} \tau_{\lambda}}+ \\
& +\frac{1}{2}\left(\tau_{\lambda}^{*}-\tau_{\lambda}\right)^{2} \frac{\mathrm{d}^{2} L_{\lambda}^{0}\left(\tau_{\lambda}, \mu\right)}{\mathrm{d} \tau_{\lambda}^{2}}+\cdots
\end{aligned}
$$

ce qui conduit, par substitution dans (7), et compte tenu de ce que les valeurs $\tau_{\lambda}$ et $\tau_{0 \lambda}-\tau_{\lambda}$, arguments d'exponentielles, ou bornes d'intégrales, peuvent être considérées comme infinies, à :

$$
q_{\mathrm{r} \lambda}(x)=-\frac{4}{3} \frac{\pi}{\chi_{\lambda}} \frac{\mathrm{d} L_{\lambda}^{0}(x)}{\mathrm{d} x} .
$$


L'introduction de la loi de Stefan conduit alors à l'expression du flux total :

$$
q_{\mathrm{r}}(x)=-\frac{16}{3} \frac{n^{2} \sigma T^{3}(x)}{\chi_{\mathrm{R}}} \frac{\mathrm{d} T(x)}{\mathrm{d} x}
$$

où :

$$
\frac{1}{\chi_{R}}=\frac{\int_{0}^{\infty} \frac{1}{\chi_{\lambda}} \frac{\partial L_{\lambda}^{0}}{\partial L^{0}} \mathrm{~d} \lambda}{\int_{0}^{\infty} \frac{\partial L_{\lambda}^{0}}{\partial L^{0}} \mathrm{~d} \lambda}
$$

définit le coefficient moyen d'absorption au sens de Rosseland, auteur à l'origine de cette pondération.

La relation (17) confirme mathématiquement l'assimilation de la propagation du rayonnement à un phénomène de diffusion, mais plus encore, est à l'origine de l'introduction de la notion de conductivité radiative :

$$
k_{\mathrm{R}}=\frac{16 n^{2} \sigma T^{3}}{3 \chi_{\mathrm{R}}}
$$

déjà citée au $\S 3$.

Une restriction s'impose cependant, qui provient de ce que doit être vérifiée l'hypothèse du milieu optiquement épais. Elle laisse prévoir tout d'abord l'impossibilité d'utiliser cette notion de conductivité radiative au voisinage immédiat des frontières. L'examen de la figure 2 montre en plus combien il est parfois délicat d'imposer globalement l'hypothèse précédente : à $1400^{\circ} \mathrm{C}$ et pour un échantillon de $10 \mathrm{~cm}$ d'épaisseur, $\tau_{\lambda}(\lambda=4 \mu)=10$, alors que $\tau_{\lambda} \quad(\lambda=2 \mu)=1$.

4.2.4 Résultats et principales caractéristiques du transfert radiatif. - Le tableau III situe nos résultats

Tableau III. - Position de nos résultats en température (transfert radiatif : $\chi=1 \mathrm{~m}^{-1} ; l=1 \mathrm{~m}$; $\left.t_{0}=300 \mathrm{~K} ; T_{1}=500 \mathrm{~K}\right)$.

[Position of our results into temperature (radiative transfer : $\chi=1 \mathrm{~m}^{-1} ; l=1 \mathrm{~m} ; t_{0}=300 \mathrm{~K}$; $\left.T_{1}=500 \mathrm{~K}\right)$.]

\begin{tabular}{|c|c|c|c|c|}
\hline$x$ & Fredholm & $\begin{array}{c}\text { CSMP } \\
\text { directions }\end{array}$ & $\begin{array}{c}\text { CSMP } \\
\text { directions }\end{array}$ & Prasad \\
\hline 1,0 & 471,31 & 470,67 & 471,14 & 471,55 \\
0,9 & 462,92 & 463,67 & 463,73 & 462,47 \\
0,8 & 455,65 & 456,52 & 456,38 & 455,44 \\
0,7 & 448,45 & 449,15 & 448,97 & 448,37 \\
0,6 & 441,10 & 441,49 & 441,37 & 440,78 \\
0,5 & 433,45 & 433,45 & 433,45 & 433,29 \\
0,4 & 425,38 & 424,95 & 425,08 & 425,21 \\
0,3 & 416,72 & 415,84 & 416,07 & 416,64 \\
0,2 & 407,21 & 405,98 & 406,17 & 406,90 \\
0,1 & 396,33 & 395,14 & 395,04 & 396,40 \\
0,0 & 381,84 & 383,02 & 382,15 & 381,38
\end{tabular}

en distribution de température et en flux par rapport à ceux de la littérature.

Si la solution de Fredholm semble en bon accord avec la littérature (écart relatif de l'ordre de $10^{-3}$ ), la comparaison avec la méthode discrétisée en direction dépend précisément du nombre des directions retenues; il apparaît ainsi bénéfique de passer de 4 à 6, l'amélioration étant particulièrement sensible aux frontières. Par ailleurs, les résultats obtenus par SINDA pour un modèle à 23 nœuds sont également en accord avec les précédents, avec un écart relatif du même ordre.

Les figures 8 et 9 montrent l'allure des températures réduites obtenues par la méthode de Fredholm [38] ainsi que celle du flux réduit

$$
Q=\frac{q_{\mathrm{r}}\left(\tau_{0}\right)}{n^{2} \sigma\left(T_{1}^{4}-T_{0}^{4}\right)}
$$

transmis entre les deux frontières.

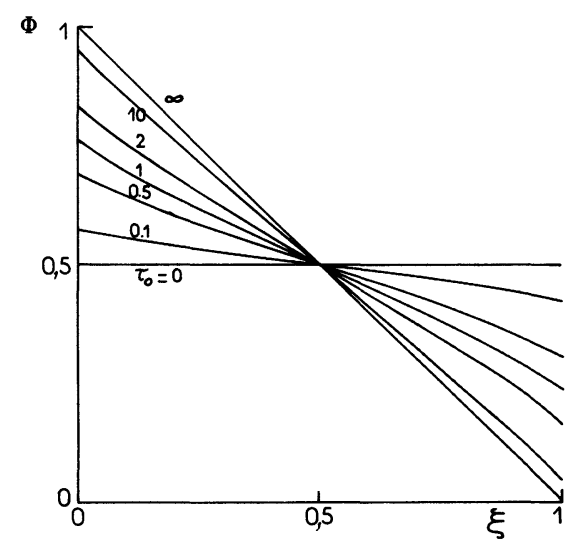

Fig. 8. - Transfert radiatif (équation de Fredholm) : champ réduit de température dans un mur fini.

[Radiative transfer (Fredholm equation). Reduced temperature field in a wall.]

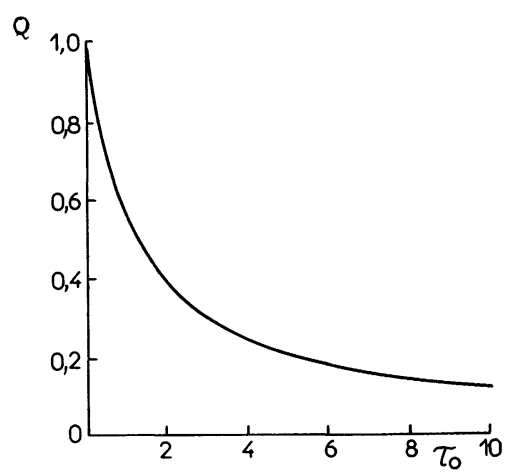

Fig. 9. - Transfert radiatif (équation de Fredholm) : évolution du flux réduit transmis en fonction de l'épaisseur optique du mur.

[Radiative transfer (Fredholm equation) : reduced transmitted flux as a function of the wall thickness.] 
Un exemple de tracé des champs de températures et de luminances est enfin présenté sur les figures 10 et 11 , correspondant au cas :

$$
\begin{gathered}
\chi=3 \mathrm{~m}^{-1} \quad l=1 \mathrm{~m} \quad T_{0}=300 \mathrm{~K} \\
T_{1}=500 \mathrm{~K} \quad n=1 \quad m=4 .
\end{gathered}
$$

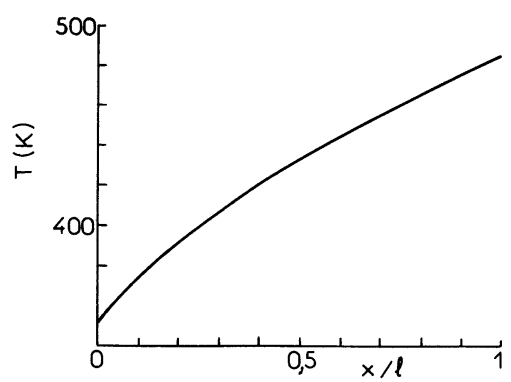

Fig. 10. - Transfert radiatif (discrétisation directionnelle des luminances) : champ de température.

[Radiative transfer (directional discretization of intensities) : temperature field.]

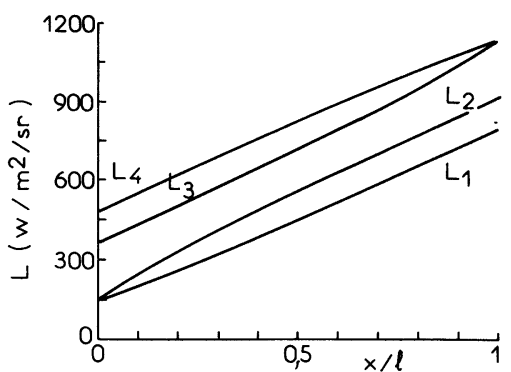

Fig. 11. - Transfert radiatif (discrétisation directionnelle des luminances) : champ des luminances directionnelles.

[Radiative transfer (directional discretization of intensities) : directional intensities field.]

Il ressort de l'examen de ces résultats un certain nombre de traits caractéristiques du transfert radiatif :

- pour des milieux très opaques, le champ $T^{4}(x)$ est linéaire en $x$,

- pour des matériaux totalement transparents, le champ en $T(x)$ est uniforme et vaut $\left[\frac{T_{1}^{4}+T_{0}^{4}}{2}\right]^{1 / 4}$,

- pour toute valeur finie de $\tau_{0}$ apparaît une discontinuité de température aux frontières et ce de façon d'autant plus marquée que $\tau_{0}$ est plus faible : une tranche infinitésimale de matériau située près de la frontière froide intègre à la fois une première information provenant de cette face, mais également, de par la transparence, une seconde provenant de la frontière chaude et de toutes les couches intermédiaires : il résulte du bilan une température locale nécessairement située entre les deux valeurs extrêmes $T_{0}$ et $T_{1}$,

- enfin, le flux échangé entre les parois s'écarte d'autant plus de $n^{2} \sigma\left(T_{1}^{4}-T_{0}^{4}\right)$, valeur obtenue dans un milieu inactif d'indice $n$, que l'épaisseur optique est élevée.

5. Le transfert couple par conduction et rayonnement. - La présence du mode conductif conduit à modifier la mise en équation par l'adjonction d'un terme supplémentaire dans le bilan énergétique. Le système se réécrit dans ces conditions :

$$
\begin{gathered}
\rho c \frac{\partial T(x, t)}{\partial t}+\operatorname{div}\left(-k \operatorname{grad} T(x, t)+2 \pi \int_{-1}^{+1} \mathbf{L}(x, \mu, t) \mu \mathrm{d} \mu\right)=0 \\
\mu \frac{\partial \mathbf{L}_{\lambda}(x, \mu, t)}{\chi_{\lambda} \mathrm{d} x}=\mathbf{L}_{\lambda}^{0}(\lambda, T(x, t))-L_{\lambda}(x, \mu, t)
\end{gathered}
$$

$\mathbf{L}(x, \mu)$, figurant dans (19) la luminance directionnelle locale totale.

De même qu'au $\S 4$ seront appliquées ici trois méthodes de résolution analytique, discrétisation directionnelle et discrétisation spatiale. De plus nous indiquerons quelques exemples de prise en compte des propriétés spectrales de l'absorption.

5.1 LA MÉTHODE ANALYTIQUE. - Elle est essentiellement commode à exploiter pour le mur gris, pour lequel l'équation (19) se transforme en :

$$
k \frac{\mathrm{d}^{2} T(\tau)}{\mathrm{d} \tau^{2}}=\frac{n^{2} \sigma}{\chi}\left[4 T^{4}(\tau)-2 T_{0}^{4} E_{2}(\tau)-2 T_{1}^{4} E_{2}\left(\tau_{0}-\tau\right)-2 \int_{0}^{\tau_{0}} T^{4}\left(\tau^{*}\right) E_{1}\left(\left|\tau-\tau^{*}\right|\right) \mathrm{d} \tau\right]
$$

Dans une première approche, Walter, Dorr et Eller linéarisent la fonction de Stefan du noyau de l'intégrale en (21), laquelle possède dans ces conditions une expression analytique simple [10]. La méthode des différences finies est alors utilisée pour aboutir à un système linéaire en $T$, résolu de façon classique (Gauss-Seidel). Cependant la linéarisation implique l'hypothèse de faibles gradients. 
Plus récemment, Grosh et Viskanta [42] ont intégré deux fois l'équation (21) par rapport à $\tau$, laquelle se transforme en :

$$
T(\tau)=G(\tau)+\frac{1}{N} \int_{0}^{\tau_{0}} F\left(\tau, \tau^{\prime}\right) T^{4}\left(\tau^{\prime}\right) \mathrm{d} \tau^{\prime}
$$

où $F$ et $G$ ont éliminé le problème de la singularité, puisque s'exprimant sur $E_{2}$ et $E_{3}$, et où $N=\frac{k \chi}{n^{2} \sigma T_{0}^{3}}$ désigne le coefficient de couplage.

Le calcul est alors achevé par une méthode itérative, qui constitue l'un des procédés couramment appliqués au traitement des équations de Fredholm (8 itérations en moyenne).

5.2 DisCRÉTISATION DIRECTIONNELLE. - Elle semble particulièrement adaptée aux matériaux non gris tels que les verres des figures 1, 2, 3, 4, dont le spectre d'absorption peut se décomposer en $\mathrm{p}$ bandes de largeur $\Delta \lambda_{\mathrm{s}}$ sur lesquelles $\chi_{\lambda}$ est supposé constant et égal à $\chi_{s}$. Le système (11), (12) devient en présence de conduction :

$$
\begin{aligned}
& \frac{\mu_{i}}{\chi} \frac{\mathrm{d} \mathbf{L}_{i s}(x)}{\mathrm{d} x}=\frac{n^{2} \sigma T^{4}(x)}{\pi} f_{\mathrm{s}}[T(x)]-\mathbf{L}_{i s} \quad \begin{aligned}
i & =1,2, \ldots, m \\
s & =1,2, \ldots, p
\end{aligned} \\
& \sum_{i, s} 2 \pi \omega_{i} \mu_{i} \frac{\mathrm{d} \mathbf{L}_{i 0}(x)}{\mathrm{d} x}-k \frac{\mathrm{d}^{2} T(x)}{\mathrm{d} x^{2}}=0 .
\end{aligned}
$$

$L_{i s}$ désignant ici les luminances directionnelles $\left(\mu_{i}\right)$ spectrales sur la bande $\left(\Delta \lambda_{\mathrm{s}}\right)$ et la fonction $f_{\mathrm{s}}[T(x)]$ la fraction d'énergie émise par le corps pour la bande $\Delta \lambda_{\mathrm{s}}$ et à la température $T(x)$ :

$$
f_{s}[T(x)]=\frac{L_{\Delta \lambda_{s}}^{0}}{\frac{n^{2} \sigma T^{4}(x)}{\pi}}=\frac{15}{\pi^{4}} \int_{\frac{h c_{0}}{n k_{\mathrm{B}} \lambda_{s} T}}^{\frac{h c_{0}}{n k_{\mathrm{B}} \lambda_{s}+1} T} \cdot \frac{u^{3}}{e^{u}-1} \mathrm{~d} u .
$$

Le système (23), (24) présente un caractère non linéaire. Anderson [43] l'a résolu par approximations successives et ce pour 10 directions et 6 bandes spectrales. Par ailleurs, nous avons obtenu la solution de ce même système dans le cas gris, en étendant au problème couplé la méthode d'optimisation appliquée au $\S 4.2 .3 .2$ au seul transfert radiatif.

5.3 Discrétisation SPatiale. - La mise à jour de l'équation (15) s'effectue tout simplement en rajoutant dans le second membre les termes de couplage conductif impliquant les conductances linéaires : $G^{l}=k S / \Delta x$, conduisant alors au système différentiel

$$
C_{i} \dot{T}_{i}=n^{2} \sigma m_{i} b_{0}\left(T_{0}^{4}-T_{i}^{4}\right)+n^{2} \sigma m_{i} b_{1}\left(T_{1}^{4}-T_{i}^{4}\right)+\sum_{j} n^{2} \sigma m_{i} m_{j}\left(T_{j}^{4}-T_{i}^{4}\right)+\sum_{j} \frac{k S}{\Delta x}\left(T_{j}-T_{i}\right) .
$$

La recherche du stationnaire du problème envisagé a été effectuée ici également à l'aide du logiciel SINDA.

Bien que n'introduisant aucune difficulté de principe, la prise en compte d'un terme de conduction pure dans (15) ne permet pas de traiter, directement du moins, le problème non gris. Celui-ci a été résolu, y compris en régime transitoire, par M. Lauriat [14] lequel approche localement (autour du point de singularité) la fonction source (laquelle est alors une fonction de Planck) par un polynôme du second degré, exprimant, au moyen d'une intégration analytique par parties, les intégrales de l'expression :

$$
\sum_{\Delta \lambda_{s}}\left\{L_{\lambda}^{0}(0) E_{2}\left(x \chi_{s}\right)+L_{\lambda}^{0}\left(l \chi_{s}\right) E_{2}\left[(l-x) \chi_{s}\right]-2 L_{\lambda}^{0}\left(x \chi_{s}\right)+\int_{0}^{l \chi_{s}} L_{\lambda}^{0}\left(x^{*} \chi_{s}\right) E_{1}\left(\left|x-x^{*}\right| \chi_{s}\right) \chi_{s} \mathrm{~d} x^{*}\right\}
$$

figurant alors dans l'écriture de la divergence du flux radiatif total, sur la base des fonctions $E_{n}(n>1)$. $\mathrm{La}$ version prédicteur-correcteur de la méthode des différences finies achève alors le calcul du champ des températures.
5.4 LES RÉSULTATS : PRINCIPALES CARACTÉRISTIQUES DU TRANSFERT COUPLÉ RAYONNEMENT-CONDUCTION. Bien que présentant une distorsion certaine, le champ des températures relatif au transfert couplé voit sa continuité assurée aux frontières par la présence du 


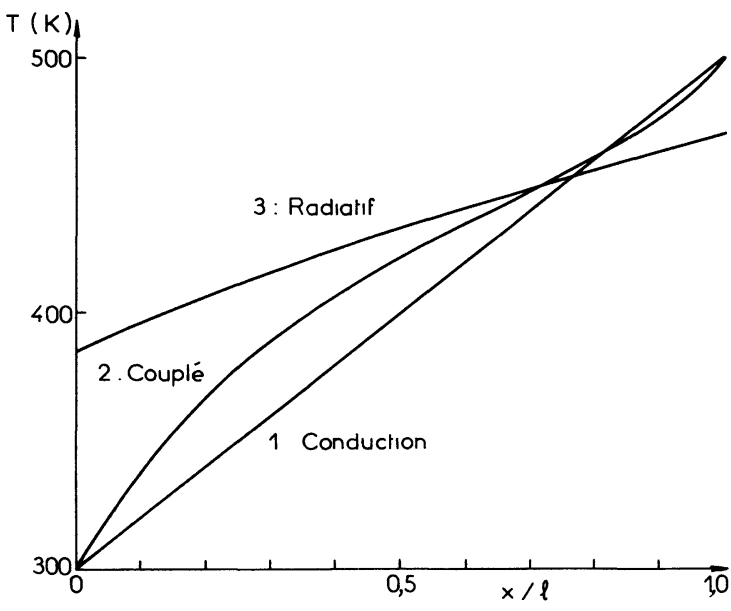

Fig. 12. - Comparaison de l'allure des champs stationnaires de température suivant le mode de transfert.

[Comparison of the steady state temperature fields corresponding to the different modes of transfer.]

mode conductif : figure $12\left(\chi=1 \mathrm{~m}^{-1}, l=1 \mathrm{~m}\right.$, $k=2 \mathrm{~W} / \mathrm{m} /{ }^{\circ} \mathrm{C}, n=1, T_{0}=300 \mathrm{~K}, T_{1}=500 \mathrm{~K}$ ) [38].

On observe également que le rayonnement a tendance à niveler les gradients lorsqu'il est pur (courbe 3 ), mais qu'en présence de conduction il les accentue près des frontières, créant un effet de puits près de la frontière chaude et un effet de source près de la frontière froide (il accentue en fait le départ d'énergie de la zone chaude vers la zone froide).

Par ailleurs, une étude très complète de l'influence des divers paramètres (nature des frontières, facteurs d'émission, conductivité phonique, épaisseur géométrique, finesse du modèle spectral) étant développée dans [14], nous résumerons ici, dans le cas du verre à vitre, les résultats provenant de la prise en compte des variations spectrales de son coefficient d'absorption.

Trois modèles ont été bâtis, à 1 bande (modèle $\mathrm{n}^{\circ} 1$, gris) à 2 bandes $\left(n^{\circ} 2\right)$ et à 3 bandes $\left(n^{\circ} 3\right)$ (Tableau IV et Fig. 13), la valeur moyenne sur une bande étant prise au sens de Planck.

Tableau IV. - Mise en place des différents modèles de spectre d'absorption du verre blanc.

[Implantation of the different spectral absorption model of white glass.]

\begin{tabular}{|c|c|c|c|}
\hline Modèle & $\begin{array}{c}\text { Bande } \\
\text { d'absorption }\end{array}$ & $\begin{array}{c}\text { Limites } \\
\text { spectrales } \\
\text { en microns }\end{array}$ & $\begin{array}{c}\text { Coefficient moyen } \\
\text { d'absorption } \\
\text { en m } \text { m }^{-1}\end{array}$ \\
\hline 1 & 1 & $1-4,4$ & 137,2 \\
\hline 2 & 1 & $1-2,7$ & 50,0 \\
\hline 3 & 2 & $2,7-4,4$ & 500,0 \\
\hline & 2 & $1-2,2$ & 9,34 \\
& 3 & $2,2-2,7$ & 29,37 \\
\hline
\end{tabular}

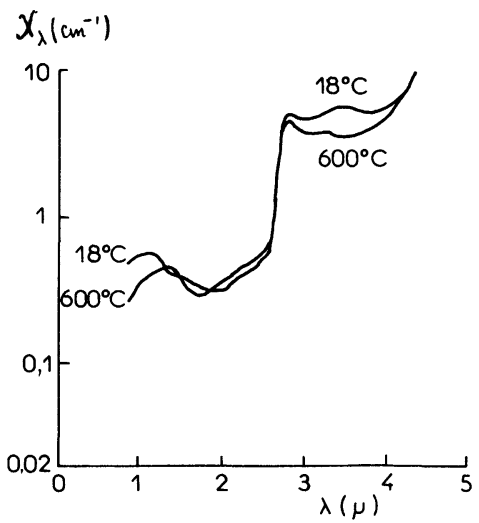

Fig. 13. - Coefficient monochromatique d'absorption du verre blanc.

[Monochromatic absorption coefficient of a white glass.]

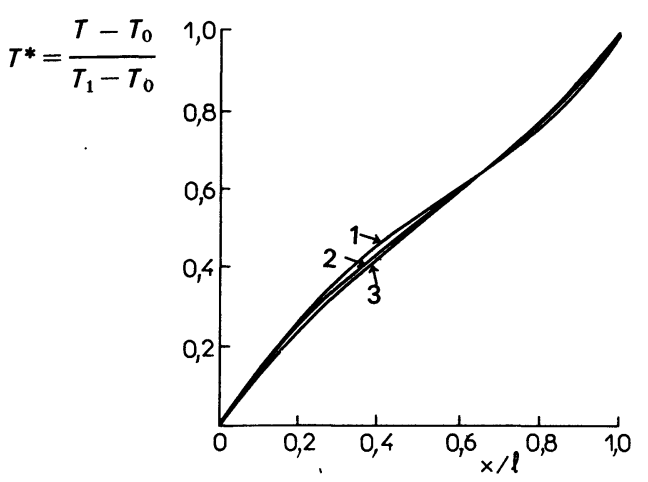

Fig. 14. - Evolution du champ réduit de température en fontion de la finesse du modèle : 1 . modèle à 1 bande spectrale; 2. modèle à 2 bandes; 3 . modèle à 3 bandes.

[The reduced temperature field as a function of the refinement of the model. 1. one band model; 2. two bands model; 3. three bands model.]

Il résulte de la figure 14 que l'affinement du modèle spectral est à l'origine d'une diminution de la distorsion, mais il n'est pas possible cependant de confondre la courbe no 3 (modèle à 3 bandes) avec un champ de pure conduction $\left(10^{\circ}\right.$ d'écart à $\left.x^{*}=0,2\right)$.

Une remarque vaut enfin d'être formulée à l'égard de la conductivité radiative : ayant tracé [38] le champ des températures et calculé celui des densités de flux, nous avons pu chiffrer à toute abscisse le quotient $k_{\mathrm{r}}^{2}$ de la densité de flux par le gradient thermique; la figure 15 montre les variations des différentes conductivités qu'il est alors possible de définir :

- conductivité phonique $k$, uniforme,

- conductivité radiative de Rosseland $k_{\mathrm{r}}^{1}$ et conductivité effective associée $k_{\mathrm{e}}^{1}=k+k_{\mathrm{r}}^{1}$,

- conductivité locale $k_{\mathrm{r}}^{2}$ et conductivité effective associée $k_{\mathrm{e}}^{2}=k+k_{\mathrm{r}}^{2}$.

Notre étude montre ainsi que dans ce cas particulier (mur gris, $\chi=1 \mathrm{~m}^{-1}, k=2 \mathrm{~W} / \mathrm{m} / \mathrm{K}$ ), le transfert calorifique n'est certainement pas assimilable à 


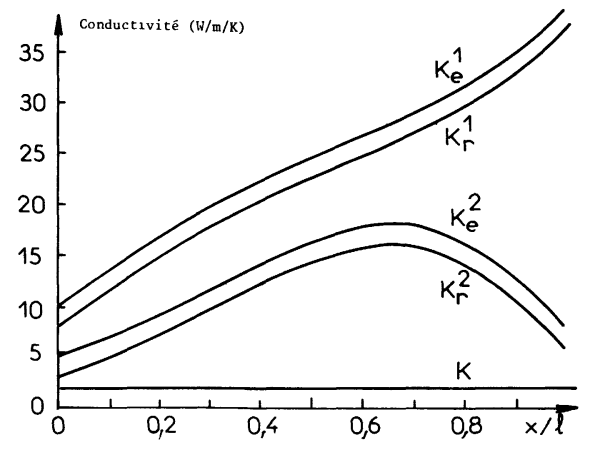

Fig. 15. - Variation spatiale des conductivités de Rosseland $k^{1}$ et locales $k^{2}$ : modèle gris, $\chi=1 \mathrm{~m}^{-1}, \lambda=2 \mathrm{~W} / \mathrm{m} / \mathrm{k}$.

[Spatial evolution of the conductivities in a gray $\operatorname{model}\left(\chi=1 \mathrm{~m}^{-1}\right.$, $\lambda=2 \mathrm{~W} / \mathrm{m} / \mathrm{k}): k^{1}:$ Rosseland, $k^{2}:$ local.]

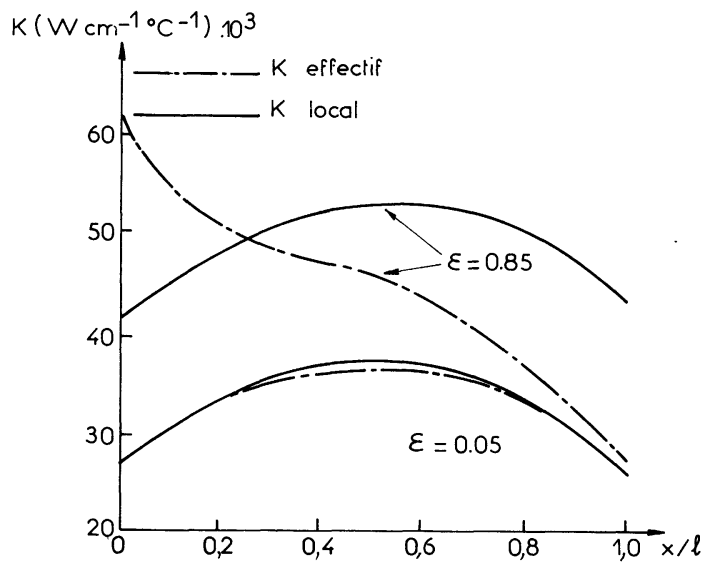

Fig. 16. - Variation spatiale des conductivités locales et effectives pour un échantillon de quartz (modèle multibande).

[Spatial evolution of the local and effective conductivities in a quartz wall (multibands model).] un phénomène de diffusion (pour lequel flux total et gradient de température locaux sont proportionnels).

Ce défaut de l'approche de Rosseland peut persister même lorsque l'on introduit dans $k_{\mathrm{r}}$ les effets de frontière (géométrie, émissivité...) ainsi que les caractéristiques spectrales du matériau, comme le montre la figure 16 [44] relative à un échantillon de quartz.

6. Conclusions. - Tout en contribuant à préciser la position actuelle du problème des transferts couplés par rayonnement et conduction, la présente note fait ressortir le caractère nouveau que présente la thermocinétique appliquée aux matériaux semitransparents, tout en réunissant par ailleurs l'essentiel des techniques numériques utilisées à l'heure actuelle dans la résolution des équations dynamiques. Il est toutefois à signaler que le gain de temps de mise en langage machine, voire en temps de calcul même, lié à une modélisation excessive tant des phénomènes eux-mêmes (assimilation du transfert radiatif à une loi de type diffusion) que de certaines propriétés (absorption spectrale grise) est parfois à l'origine d'écarts sensibles dans les résultats. Par ailleurs, l'exploitation des moyens de calculs récents que constituent les logiciels permet d'envisager sous un jour nouveau ces problèmes complexes des transferts combinés, tant en régime stationnaire que variable. Plus encore, les ressources (optimisation, filtre de Kalman, ...) offertes par ces outils récents ouvrent la voie non seulement à l'analyse de ces phénomènes mais également à d'éventuels traitements d'identification de paramètres thermophysiques concernant les échanges couplés par rayonnement et conduction.

\section{Bibliographie}

[1] Coenen, M., Transparence d'un bain coloré. Glastech. Ber. 41 (1968) $1-10$.

[2] Men, A. A., Sergueev, O. A., Le transfert de chaleur conductif par rayonnement dans les matériaux solides semi-transparents. Int. J. Heat Mass Transfer 15 (1972) 1807-1817.

[3] Fourier, J., Théorie analytique de la chaleur, 1808, 2e édition F. Didot (Paris) 1822.

[4] SChuster, A., The influence of radiation on the transmission of heat (The London, Edimburgh and Dublin) Philos. Mag. Sci. V (January-June 1903) sixth serie.

[5] Siegel, R., Howell, J., Thermal Radiation Heat Transfer. NASA-SP-164, 1968 (I), 1969 (II), 1971 (III), Washington.

[6] Preston, F. W., The meaning of the term diathermancy and the heating of glass in tank furnaces. J. Soc. Glass. Technol. 31 (1947) $134-140$.

[7] Czerny, M., Genzel, L., Energiefluss und Temperaturverlauf im Glasbad von Schmelzwannen als Folge von Wärmeleitung und Wärmestrahlung. Glastech. Ber. 25 (1952) 387392.

[8] Geffrken, W., Zur Vorleitung der Wärme in Glas bei hohen Temperaturen I. Glastech. Ber. 25 (1952) 392-396.

[9] GeffCKen, W., Zur Vorleitung der Wärme in Glasbeihohen Temperaturen II. Glastech. Ber. 29 (1965) 42-49.

[10] Walter, A., Dörr, J., Eller, E., Mathematische Berechnung der Temperaturverlauf in der Glasschmelze mit Berück- sichtigung von Wärmeleitung und Wärmestrahlung. Glastech. Ber. 26 (1953) mai.

[11] Poltz, H., Die Wärmeleitfahigkeit von Flussigkeiten. Int. J. Heat Mass Transfer 5 (1962) 307-316. 8 (1965) 515-527, 8 (1965) 609-620, 10 (1967) 1075-1088.

[12] Chui, K., Gardon, R., Interaction of radiation with conduction in glass. J. Am. Ceram. Soc. 52 (1968) no 10.

[13] Anderson, E. E., Viskanta, R., Stevenson, W. H., Heat Transfer through semitransparent solids. J. Heat Transfer 176-186 (1973) May.

[14] Lauriat, G., Contribution à l'étude du transfert de chaleur dans les milieux semi-transparents. Thèse de $3^{\mathrm{e}}$ Cycle, Paris, 1974.

[15] GenZEL, L., Measurement of infrared absorption of glass between $20^{\circ} \mathrm{C}$ and $1360^{\circ} \mathrm{C}$. Glastechn. Ber. 24 (1951) 55-63.

[16] Neuroth, N., Der Einfluss der Temperatur auf die spektrale Absorption von Gläsern in Ultraroten I. Glastechn. Ber. 25 (1952) 242-249.

[17] Neuroth, N., Der Einfluss der Temperatur auf die spektrale Absorption von Gläsern in Ultraroten II. Glastechn. Ber. 26 (1953) 66-69.

[18] Grove, F. J., Jellyman, P. E., The infrared transmission of glass in the range room temperature to $1400^{\circ} \mathrm{C}$. J. Soc. Glass Technol. 39 (1955) 3-15. 
[19] Blazek, A., Endrys, J., Kada, J., Stanek, J., Strahlungswärmeleitfähigkeit von Glas. Einfluss der Glaszusammensetzung auf seine Wärmedurchlässigkeit. Glastechn. Ber. 49 (1976) 75-81.

[20] WedDing, B., Measurements of high temperature absorption coefficient of glass. J. Am. Ceram. Soc. 58 (1975) 102-105.

[21] Traverse, J. P., Ades, C., Naudin, F., Propriétés optiques des oxydes dissous dans le verre fondu. Compte rendu de fin d'étude, D.G.R.S.T., 1977.

[22] VAN ZEe, A. F., BabCock, C. L., A method for measurement of thermal diffusivity of molten glass. J. Ceram. Soc. 34 (1951) 244-250.

[23] Grove, F. J., Charnok, H., Die Wärmeleitung im geschmolzenen Glas; ein Vergleich zwischen Theorie und Versuchergebnissen. Glastechn. Ber. 32 (1959) 24-28.

[24] Lajarte, M., BrÉ, M., Kermabon, P., Diffusivité thermique de quelques verres industriels aux températures élevées. $8^{\mathrm{e}}$ Congrès International du Verre, juin 1968.

[25] KröGer, C., Elighausen, H., Ueber das Wärmeleitvermögen des einschmelzenden Glasmenges Glastechn. Ber. 32 (1959) 362-373.

[26] ECKHARDT, G., Wärmeleitung und Wärmestrahlung in Gläsern zwischen $100^{\circ} \mathrm{C}$ und $1200^{\circ} \mathrm{C}$. Glastechn. Ber. 32 (1959) $373-380$.

[27] Champomier, F., Mesure de la conductivité et de la diffusivité thermiques d'un verre float entre $0^{\circ} \mathrm{C}$ et $550{ }^{\circ} \mathrm{C}$ environ. Rapport interne St-Gobain, 1976

[28] BrÉ, M. Kermabon, P., Mesure de la diffusivité thermique globale des verres jusqu'à $1400^{\circ} \mathrm{C}$. Verres Réfract. 23 $\mathrm{n}^{\circ} 9$ (1969) 3-9.

[29] Neuroth, N., Zusammenstellung einiger Messergebnisse der Wärmeleitfahigkeit von Glasschmelzen. Glastechn. Ber. 32 (1959) 197-198.

[30] Gardon, R., Some Heat Transfer problems in glass technology. Soc. Glass Technol. (1969) 85-95.

[31] Anderson, E. E., VisKanta, R., Effective Thermal conducti- vity of heat transfer through semi-transparent solids. J. Amer. Ceram. Soc. 56 (10) (1973) 541-546.

[32] Eyrou, ND. Glicksman, L. R., An experimental and analytical study of radiative and conductive heat transfer in molten glass. J. Heat Transfer (1972) May.

[33] Chupp, R., Development and experimental evaluation of a technique to determine the temperature distribution in semi-transparent solids, from remotely sensed spectral emission data. Ph. D. Purdue University, 1973.

[34] Schatzman, E., Pecker, J. C., Astrophysique générale (Masson, Paris) 1959.

[35] Delner, T. N., Vulliet, W. C., Conditions for local thermodynamic equilibrium in uranium. NASA-SP-236, 1970.

[36] Sparrow, E. M., Cess, R. D., Radiation Heat Transfer. Brooks./Cole, Belmont California, 1970.

[37] Krishna Prasad, K., Hering, R. G., Numerical integration of a nonlinear singular differential equation. J. Comput. Phys. 6 (1970) 406-416.

[38] Saulnier, J. B., Ferrante, J. G., Bouchez, J. P., Thermocinétique des transferts couplés radiatifs et conductifs. Partie 1 : Analyse par SINDA et CSMP. Mars 1978, ESA Journal.

[39] Chandrasekhar, S., Radiative Transfer. Dover Publication Inc., New York, 1960.

[40] SCHEID, F., Theory and Problems of numerical analysis, Schaum's outline series. Mac Graw-Hill Company, 1968.

[41] Hottel, H. C., SARofim, A. F., Radiative Transfer. Mac GrawHill Book Company, 1967.

[42] Viskanta, R., Grosh, R. J., Heat Transfer by simultaneous conduction and radiation in an absorbing medium. J. Heat Transfer, Feb. (1962) 63-72.

[43] Anderson, E. E., Viskanta, R., Spectral and boundary effects on coupled conduction-radiation heat transfer through semi-transparent solids. Wärme und Staffübertragung 1 (1974) 14-24.

[44] Viskanta, R., ANDerson, E. E., Heat Transfer in semitransparent solids. Academic Press. Adv. Heat Transfer, 11 (1975) 317-441. 